\title{
EFFECT OF FEEDING DIFFERENT LEVELS OF YELLOW CORN WITH OR WITHOUT MULTI-ENZYMES OR PREBIOTIC SUPPLEMENTATION ON GROWTH PERFORMANCE AND ECONOMICAL EFFICIENCY IN
} GROWING RABBIT RATIONS

\author{
Tork M. I. Dorra ${ }^{1}$; Hayam M. Abo El-Maaty ${ }^{1}$; Gihan M. El Moghaz ${ }^{2}$ and Rana H. E. Eid ${ }^{2}$ \\ ${ }^{1}$ Department of Poultry Production, Faculty of Agriculture, Mansoura University, Egypt. \\ ${ }^{2}$ Regional Center for Food and Feed, Agricultural Research Center, Giza, Egypt.
}

(Received 19/12/2017, accepted 4/2/2018)

\section{SUMMARY}

\begin{abstract}
$\mathrm{O}$ ne hundred and eight, 6 weeks of age weaning New Zealand White (NZW) rabbits were used in this study to investigate the impact of feeding three levels of yellow corn diets without or with enzymes or prebiotics or both. Rabbits were distributed in a completely randomized experimental design in a $3 \times 4$ factorial arrangements, with the variables being supplementation or not with enzymes (500 g / ton feed) or prebiotics (500 g / ton feed) or both $(500 \mathrm{~g}$ enzymes $+500 \mathrm{~g}$ prebiotics $) /$ ton feed. The experimental groups of rabbits were fed their respective experimental rations in pelleted form. Three basal rations were used in this study; the first containing 5\% corn (R1), the second one contained 10\% corn (R5) and the third one contained 15\% corn (R9). The composition and calculated analysis of the basal diets were done according to NRC (1977). The basal ration (R1) was supplemented with enzymes or prebiotics or both for R2, R3 and R4, respectively. While the basal ration (R5) was supplemented with enzymes or prebiotics or both for R6, R7 and R8, respectively, and the basal ration (R9) was supplemented with enzymes or prebiotics or both for R10, R11 and R12, respectively. Apart from the effect of feed rations which were contained 5\% or $15 \%$ corn, were significantly $(\mathrm{P}<0.05)$ higher live body weight $(\mathrm{LBW})$ of rabbits 7 weeks of age than feeding on ration was contained $10 \%$ corn, but without significant effect on LBW at different ages of the experimental period, from 8 to 15 weeks of age. Regardless of the effect of dietary corn level, addition of enzymes or prebiotics or both had no significant effect $(\mathrm{P}>0.05)$ on live body weight of rabbits at different ages of the experimental period, from 6 to 15 weeks of age. Apart from the effect of corn levels and dietary supplements had no significant effect $(\mathrm{P}<0.05)$ on daily feed intake $(\mathrm{DFI})$ of rabbits during the period from 6 to 15 weeks. The daily feed intake (DFI) of rabbits fed rations contained $5 \%, 10 \%$ and $15 \%$ corn were $89.88,89.03$ and $89.62 \mathrm{~g} / \mathrm{d}$, respectively. The interaction between dietary corn levels and added supplements on feed conversion ratio (FCR) during the whole experimental period (from 6-15 weeks) showed that, the FCR of the growing rabbits were better $(\mathrm{P}<0.05)$ with rations $\mathrm{R} 8$, R9 and $\mathrm{R} 11$ (3.93, 4.07 and $4.04 \mathrm{~g} / \mathrm{d}$, respectively). The effect of dietary interaction, showed that addition of enzymes with diet containing 5\% corn or diet containing $10 \%$ corn without supplements lead to a significant $(\mathrm{p}<0.05)$ increased in weight of viscera. The economic efficiency $(\%)$ showed that feeding growing rabbits on ration contained $10 \%$ corn and supplemented with enzymes plus prebiotics (R8) or feeding diet contained $15 \%$ corn without supplements (R9), improved of feed efficiency (102.8 and $102.4 \%$, respectively).
\end{abstract}

Keywords: performance, multi-enzymes, prebiotic and economic efficiency.

\section{INTRODUCTION}

Rabbit meat is excellent protein quality, low in total as well as saturated fat, cholesterol and sodium. Therefore, rabbit production is considered as a good source of meat in tropical developing countries where there is an abundance of by- product feedstuffs (Taylor et al. 1989). Several studies carried out on poultry and pigs have shown that the nutritive value of feedstuffs, especially of poor quality, can be enhanced by supplemental enzymes (Cowan et al.1996). Abo El-Maaty et al. (2014) were designed an experiment to study the effect of partial or complete substitution of cucumber vines straw (CVS) for dietary clover hay in presence or absence of multi-enzyme addition on growth performance of New Zealand White (NZW) rabbits. They found that, dietary supplementation with enzyme preparation (Natuzyme, NZ) to the diet containing $30 \% \mathrm{CVS}$ restored the normal values of growth performance and economic efficiency attained by the control rabbits. 


\section{Dorra et al.}

On the other hand, probiotics such as yeast culture can be used to manipulate rumen fermentation in ruminant (Williams and Newbold, 1990). Rabbits may also benefit from prebiotic feed additives as they are highly dependent on hindgut fermentation to utilize their feed efficiently. However the use of such feed additives in the rabbit has not been extensively studied (Shanmuganathan et al. 2004).

The important carbohydrates in rabbit feed are starch (digestible) and fiber (indigestible). Starch provides energy that is readily available and easily digestible. Although grains are good sources of starch, rabbit diets high in grain can cause a starch overload in the hindgut leading to enteritis. Fiber is important for overall gut health and mobility and appetite stimulation. The bacterial population in the hindgut allows rabbits to diets fiber to some extent. Rabbits have a high feed intake (65- $80 \mathrm{~g} / \mathrm{kg}$ body weight) and a rapid feed transit time (about 19 hours) and this are able to consume lower quality forages and still meet their nutritional needs (De Blac and Wiseman, 2003). The benefits of adding enzymes to diets of nonruminant animals particularly poultry, has become more important (Campell and Bedford, 1992). These enzymes potentially hydrolyze non soluble protein (NSP), reduce the viscosity of gut contents, and result in improvements in nutrient absorption. Dietary fiber plays an important role in the diet of rabbit because of its influence on caecal microbial activity (Gidenne et al. 2010a).

The optimal dietary fiber level for growing rabbits is variable and may depend largely upon other factors such as type of fiber, age and breed of rabbits, and /or digestible energy content of the diet (Gidenne et al. 2010). Most organisms used in probiotics are strains of gram positive bacteria of the genera [bacillus (B. subtilis), enterococcus (E. faecium), lactobacillus (l. acidophilus), Bifid bacteria (B. lactic ), streptococcus (S.infanarius) and some yeasts or fungi such as Saccharomyces cerevisae]. The use of oligosaccharides to affect increase in gut bacteria seen as beneficial has been for many years; however the term "prebiotic" was only first coined in mid-1990 (Gibson and Roberfroid, 1995). This clearly built upon the success of probiotics for micro flora management approaches.

The present study was performed to investigate the influence of feeding diets without or with enzymes or prebiotics or both on the performance, carcass traits and economic efficiency of growing New Zealand white rabbits.

\section{MATERIALS AND METHODS}

The experimental work of the present study was carried out at the Poultry Research Unit, Agricultural Research and Experimental Center, Faculty of Agriculture, Mansoura University.

\section{Experimental animals and management}

One hundred and eight, 6 weeks old weaning New Zealand White (NZW) rabbits were used in this study. Rabbits were randomly distributed into 12 equal experimental groups; each contained three equal replications. Each replicate group ( 3 rabbits) was housed in a separate cage with the following dimensions $50 \times 50 \times 45 \mathrm{~cm}$ for length, width and height, respectively. Rabbits were fed their respective experimental diets from 6 to 15 weeks of age. Feed and water were offered ad libitum throughout the experimental period. The values of live body weight and feed intake were recorded on a replicate group basis and thus daily weight gain and feed conversion were also calculated.

\section{Feed supplements}

In the present study, the multi-enzyme extra and prefect were performance in aquaculture and agriculture (KIOTECHAGIL). The specific wall stabilized enzymes combination of German origin (xylanase, betaglucanase, alpha amylase, protease and phytase), which $500 \mathrm{~g}$ were added to ton feed of the basal diet. Prefect is a buffered blend of specific acids on a unique mineral carrier system combined with a fructo-oligosaccharide (FOS) to promote a healthy gut microflora, which $500 \mathrm{~g}$ were added to ton feed of the basal diet.

\section{Experimental rations and design}

An experiment $(3 \times 4)$ was conducted with factorial arrangement of treatments, being three levels of yellow corn without or with enzymes or prebiotics or both. Thus, twelve experimental rations were formulated and used. The experimental groups of rabbits were fed their respective experimental rations in pelleted form. Three basal rations were used in this study; the first containing 5\% corn (R1), the second one contained $10 \%$ corn (R5) and the third one contained $15 \%$ corn (R9). The composition and calculated analysis of the basal diets are presented in Table 1, according to NRC (1977). 
The basal ration (R1) was supplemented with enzymes or prebiotics or both for R2, R3 and R4, respectively. While the basal ration (R5) was supplemented with enzymes or prebiotics or both for R6, R7 and R8, respectively, and the basal ration (R9) was supplemented with enzymes or prebiotics or both for R10, R11 and R12, respectively.

\section{Growth performance parameters}

Live body weights of the experimental rabbits were individually recorded at the start of the experiment and at a weekly basis thereafter and were estimated to the nearest five grams in the early morning before receiving any feed or water. Body weight gain and feed conversion ratio were also calculated on a replicate group basis. Mortality of rabbits was also monitored and recorded daily.

Table (1): Ingredients and chemical composition of the basal diets.

\begin{tabular}{|c|c|c|c|}
\hline \multirow[b]{3}{*}{ Feed ingredient } & \multicolumn{3}{|c|}{ Basal diets } \\
\hline & (1) R1 & (2) R5 & (3) R9 \\
\hline & $5 \%$ yellow corn & $10 \%$ yellow corn & $15 \%$ yellow corn \\
\hline Barley & 12 & 10 & 9 \\
\hline Yellow corn & 5 & 10 & 15 \\
\hline Wheat bran & 35 & 30 & 25 \\
\hline Soybean meal & 7.5 & 8.5 & 9.5 \\
\hline Alfalfa hay & 35.7 & 36.7 & 36.7 \\
\hline Molasses & 2.0 & 2.0 & 2.0 \\
\hline Limestone & 1.0 & 1.0 & 1.0 \\
\hline Dicalcium phosphate & 1.2 & 1.2 & 1.2 \\
\hline Sodium chloride & 0.3 & 0.3 & 0.3 \\
\hline Vit. \& Min. premix & 0.3 & 0.3 & 0.3 \\
\hline Total & 100 & 100 & 100 \\
\hline \multicolumn{4}{|c|}{ Calculated analysis on DM basis (NRC, 1977) } \\
\hline Digestible energy (Kcal/kg) & 2597 & 2600 & 2615 \\
\hline Crude protein $(\%)$ & 16.02 & 16.08 & 16.09 \\
\hline Crude fiber $(\%)$ & 14.37 & 14.22 & 13.84 \\
\hline Ether extract $(\%)$ & 2.84 & 2.83 & 2.81 \\
\hline Calcium (\%) & 1.24 & 1.25 & 1.24 \\
\hline Total phosphorus $(\%)$ & 0.77 & 0.74 & 0.70 \\
\hline Lysine (\%) & 0.70 & 0.71 & 0.72 \\
\hline Methionine (\%) & 0.19 & 0.20 & 0.20 \\
\hline Methionine+cysteine $(\%)$ & 0.50 & 0.50 & 0.51 \\
\hline
\end{tabular}

*Premix: Each $2 \mathrm{~kg}$ of the premix contained Vit A, $10.000000 \mathrm{IU}$; Vit D, $2000000 \mathrm{IU}$, Vit E, $10000 \mathrm{mg}$; Zinc, 3000 mg, Manganese, $2000 \mathrm{mg}$; Iron, $4000 \mathrm{mg}$; Copper, $1000 \mathrm{mg}$; Iodine, $100 \mathrm{mg}$; Selenium, $10 \mathrm{mg}$; Cobalt, $10 \mathrm{mg}$; Sodium, $23000 \mathrm{mg}$; and Magnesium, $2000 \mathrm{mg}$; ( $\mathrm{CaCo}_{3}$ was added to $2.0 \mathrm{~kg}$ ).

\section{Carcass evaluation}

At the end of the experimental period, three rabbits from each experimental treatment were randomly chosen and slaughtered to study carcass characteristics. Rabbits were fasted for approximately 18 hours before slaughtering, individually weighed and slaughtered according to the rules of Islamic religion. Slaughter data were immediately recorded for the individual rabbits. Skinning was carried out by removing the skin including tail and legs. Carcasses were eviscerated and the different organs (e.g. liver, heart, kidneys, viscera and lungs) were removed and immediately weighed to the nearest gram. Absolute weights of dressed carcass and organs and dressing-out percentage were estimated.

\section{Economic efficiency}

The local price of daily body weight gain and daily feed cost were calculated depending on the prevailing prices being: Price of $\mathrm{kg}$ body weight $=35.00 \mathrm{LE} ; \mathrm{Kg}$ enzymes $=150 \mathrm{LE}$; Kg prebiotics $=220$ $\mathrm{LE} ; \mathrm{Kg}$ feed $(\mathrm{R} 1)=4.2 \mathrm{LE} ; \mathrm{Kg}$ feed $(\mathrm{R} 2)=4.28 \mathrm{LE} ; \mathrm{Kg}(\mathrm{R} 3)=4.31 \mathrm{LE} ; \mathrm{Kg}(\mathrm{R} 4)=4.39 \mathrm{LE} ; \mathrm{Kg}(\mathrm{R} 5)=$ 4.21 LE; Kg $(\mathrm{R} 6)=4.29 \mathrm{LE} ; \mathrm{Kg}$ feed $(\mathrm{R} 7)=4.32 \mathrm{LE} ; \mathrm{Kg}$ feed $(\mathrm{R} 8)=4.4 \mathrm{LE} ; \mathrm{Kg}$ feed $(\mathrm{R} 9)=4.24 \mathrm{LE} ; \mathrm{Kg}$ feed $(\mathrm{R} 10)=4.32 \mathrm{LE} ; \mathrm{Kg}$ feed $(\mathrm{R} 11)=4.35 \mathrm{LE}$ and $\mathrm{Kg}$ feed $(\mathrm{R} 12)=4.43 \mathrm{LE}$. 


\section{Dorra et al.}

- $\quad$ Total feed cost $=$ Average feed intake $(\mathrm{kg}) \times$ price per $(\mathrm{kg})$ feed.

- Weight gain price $=$ Average weight gain $(\mathrm{kg}) \times$ price per $\mathrm{kg}$ live body weight

- $\quad$ Net revenue or profit $(\mathrm{LE})=$ Price of weight gain - price of feed cost.

- $\quad$ Economic efficiency $=$ Profit $($ LE $) \times 100 /$ total feed cost.

Statistical analysis

Statistical analysis of data was carried out using the General Linear Model Program of SAS (2000). Differences among means of treatments were identified by Duncan's Multiple Range Test (Duncan, 1955). The obtained data for productive traits of different groups of rabbits were subjected to factorial analysis of variance according to the following mathematical model: $Y_{i j k}=\mu+T_{i}+L_{j}+T_{i j}+e_{i j k}$

Where; $Y_{i j k}=$ observation of the tested factor, $\mu=$ overall mean, $T_{i}=$ the effect of corn level, $L_{j}=$ the effect of supplements, $\mathrm{TL}_{\mathrm{ij}}=$ the interaction between corn level and supplements and $\mathrm{e}_{\mathrm{ijk}}=$ experimental random error.

\section{RESULTS AND DISCUSSION}

\section{Live body weights and weight gain of growing rabbits}

The effects of feeding diets containing three levels of yellow corn without or supplemented with enzymes or prebiotics or both on live body weight and weight gain of growing rabbits are presented in Tables (2 and 3). No deaths of rabbits occurred during the duration of this study.

Apart from the effect of feed rations contained $5 \%$ or $15 \%$ corn, were significantly $(\mathrm{P}<0.05)$ higher live body weight (LBW) of rabbits 7 weeks of age than those fed ration contained $10 \%$ corn (Table 2), but without significant effect on LBW at different ages of the experimental period, from 8 to 15 weeks of age. Regardless of the effect of dietary corn level, addition of enzymes or prebiotics or both had no significant effect $(\mathrm{P}>0.05)$ on live body weight of rabbits at different ages of the experimental period, from 6 to 15 weeks of age. The interaction between corn levels and supplements did not significantly affect live body weight of growing rabbits throughout the whole experimental period from 6 to 15 weeks of age.

As regards the effects of dietary treatments, rabbits fed the diets containing $5 \%$ or $15 \%$ corn exhibited significantly higher $(\mathrm{P}<0.05)$ body weight gain during the $7^{\text {th }}(6-7 \mathrm{wk})$ and $10^{\text {th }}(9-10 \mathrm{wk})$ weeks than feeding on ration was contained $10 \%$ corn (Table 3). Apart from the effect of addition of enzymes together with prebiotics had significant effect $(\mathrm{P}<0.05)$ on body weight gain $(\mathrm{BWG})$ of rabbits at $9^{\text {th }}(8-9$ $\mathrm{wk}$ ) and $12^{\text {th }}$ (11-12 wk) weeks than feeding on the control ration. At period from 9-10 weeks of age in which the control or adding prebiotics were significantly higher $(\mathrm{P}<0.05)$ body weight gain compared with those adding enzymes or enzymes with prebiotics. Dietary treatments by added supplements and corn levels interaction did not significantly affect live body weight of growing rabbits throughout the whole experimental period.

Xiccato et al. (1998) reported that when high starch diets were fed to rabbits older than $35 \mathrm{~d}$ of age under more balanced nutritional conditions and respecting minimum fiber level, no significance recorded on health status. Similarly, Nizza and Moniello (2000) did not report any effect of the starch source on growth performance and meat properties.

Several works have already investigated the efficacy of digestive enzymes in 35 to 49 day old of rabbits (Remois et al. 1996). The results of these studies have shown little or no effects of enzyme supplementation on fattening rabbit performance. However, in younger animals from 25 to 39 days of age, addition enzymes has improved daily gain by $+3.1 \%$. Danicke et al. (1999) suggested that addition of b-xylanases could modulate gut microflora, enhancing growth performance and health status of the animals, especially in the post - weaning period. Falcao-e-Cunha et al. (2007) did not detect any significant effect of enzymes on rabbit's performance. Garcia et al. (2005) found some positive results with proteases and proteases + xylanases.

The effects of feeding diets containing three levels of yellow corn without or supplemented with enzymes or prebiotics or both on daily feed intake (DFI) and feed conversion ratio (FCR) of growing rabbits are presented in Tables 4 and 5, respectively. Apart from the effect of corn levels and dietary supplements had no significant effect $(\mathrm{P}<0.05)$ on DFI of rabbits during the periods of 9-10, 10-11, 11$12,12-13,13-14,14-15$ weeks of age (Table 4). The DFI of rabbits fed rations contained 5\%, $10 \%$ and 
$15 \%$ corn were $89.88,89.03$ and $89.62 \mathrm{~g} / \mathrm{d}$, respectively. The DFI without supplement $0.0 \%$ enzyme (B1) or with $0.05 \%$ enzyme (B2) or with $0.05 \%$ prebiotic (B3) or with $0.05 \%$ enzyme $+0.05 \%$ prebiotic (B4) were 88.67, 89.04, 90.14 and $90.19 \mathrm{~g} / \mathrm{d}$, respectively, (Table 4).

Table (2): Effect of fed diets containing three levels of corn supplemented with enzyme or prebiotic or both on live body weight of growing rabbits from 6 to 15 weeks of age.

\begin{tabular}{|c|c|c|c|c|c|c|c|c|c|c|}
\hline \multirow{3}{*}{ Dietary treatment } & \multicolumn{10}{|c|}{ Live body weight (g) } \\
\hline & \multicolumn{10}{|c|}{ Week } \\
\hline & 6 & 7 & 8 & 9 & 10 & 11 & 12 & 13 & 14 & 15 \\
\hline \multicolumn{11}{|l|}{ Corn levels \% (A): } \\
\hline $5 \%(\mathrm{~A} 1)$ & 679.7 & 809.3 & 927.1 & 1099. & 1296. & 1451. & 1609. & 1776. & 1891. & 2044. \\
\hline $10 \%(\mathrm{~A} 2)$ & 679.9 & 786.9 & 928.0 & 1097. & 1275. & 1444. & 1595 . & 1757. & 1889. & 2057. \\
\hline $15 \%(\mathrm{~A} 3)$ & 684.0 & 807.6 & 932.6 & $\hat{1} 111$. & $\overline{1} 295$. & 1458. & 1625. & 1773. & $\overline{1} 906$. & $20 \overline{6} 64$. \\
\hline SEM & 3.35 & 5.84 & 9.05 & 9.66 & 12.92 & $\overline{1} 3.55$ & $\overline{1} 4.92$ & 19.00 & 22.16 & 27.25 \\
\hline Significance & NS & $*$ & NS & NS & NS & NS & NS & NS & NS & NS \\
\hline \multicolumn{11}{|l|}{ Supplements (B): } \\
\hline $0.0 \%(\mathrm{~B} 1)$ & 682.2 & 805.7 & 929.3 & 1088. & 1284. & 1442. & 1593. & 1743. & 1870. & 2035. \\
\hline $0.05 \% \mathrm{E}(\mathrm{B} 2)$ & 677.2 & 791.9 & 923.7 & $\overline{1} 094$. & 1278. & 1444. & $\overline{1} 596$. & $\overline{1} 748$. & 1882. & 2046. \\
\hline $0.05 \% \mathrm{P}(\mathrm{B} 3)$ & 681.7 & 802.3 & 930.7 & 1104. & 1298. & 1465. & 1624. & 1792. & 1910. & 2069. \\
\hline $0.05 \% \mathrm{E}+0.05 \%$ & 683.9 & 805.2 & 933.3 & $\hat{1} 123$. & 1295. & 1454. & 1626. & 1790. & 1920. & 2070. \\
\hline SEM & 3.86 & 6.74 & 10.45 & 11.16 & $\overline{1} 4.93$ & 15.64 & 17.22 & $\hat{2} 1.94$ & 25.58 & $\overline{3} 1.95$ \\
\hline Significance & NS & NS & NS & NS & NS & NS & NS & NS & NS & NS \\
\hline \multicolumn{11}{|l|}{ AB Interaction: } \\
\hline $\mathrm{A} 1 \mathrm{~B} 1=\mathrm{R} 1$ & 671.6 & 790.5 & 892.8 & 1055. & 1267. & 1411. & 1546. & 1702. & 1814. & 1982. \\
\hline $\mathrm{A} 1 \mathrm{~B} 2=\mathrm{R} 2$ & 685.0 & 724.5 & 951.1 & $\overline{1} 130$. & 1323. & $\overline{1} 479$. & 1929. & 1795. & 1923. & 2094. \\
\hline $\mathrm{A} 1 \mathrm{~B} 3=\mathrm{R} 3$ & 685.0 & 814.4 & 942.8 & 1103. & 1291. & 1452. & 1623. & 1804. & 1909. & 2057. \\
\hline $\mathrm{A} 1 \mathrm{~B} 4=\mathrm{R} 4$ & 677.2 & 807.8 & 921.7 & $\hat{1} 107$. & $\overline{1} 303$. & 1463. & 1637. & 1802. & 1918. & 2042. \\
\hline $\mathrm{A} 2 \mathrm{~B} 1=\mathrm{R} 5$ & 687.2 & 800.0 & 942.8 & 1097. & 1285. & $\overline{1} 447$. & 1592. & 1746. & 1875. & 2040. \\
\hline $\mathrm{A} 2 \mathrm{~B} 2=\mathrm{R} 6$ & 675.0 & 762.4 & 909.5 & 1076. & 1260. & 1442. & 1586. & $\overline{1} 732$. & 1871. & 2032. \\
\hline $\mathrm{A} 2 \mathrm{~B} 3=\mathrm{R} 7$ & 678.3 & 789.7 & 929.2 & $\overline{1} 102$. & 1292. & 1463. & $\overline{1} 606$. & 1776. & $\overline{1} 895$. & 2051. \\
\hline $\mathrm{A} 2 \mathrm{~B} 4=\mathrm{R} 8$ & 678.9 & 795.6 & 930.6 & $\tilde{1} 111$. & 1265 . & 1425 . & 1597. & 1772. & 1917. & 2105. \\
\hline $\mathrm{A} 3 \mathrm{~B} 1=\mathrm{R} 9$ & 687.2 & 826.7 & 952.2 & 1113. & 1302. & 1466. & 1641. & 1782. & 1921. & 2082 . \\
\hline $\mathrm{A} 3 \mathrm{~B} 2=\mathrm{R} 10$ & 671.7 & 788.9 & 910.6 & 1076. & 1252. & $\overline{1} 411$. & $\overline{1} 572$. & 1718. & 1852. & 2013. \\
\hline $\mathrm{A} 3 \mathrm{~B} 3=\mathrm{R} 11$ & 681.7 & 802.8 & 920.0 & $\overline{1} 106$. & 1310. & 1481. & 1642. & 1796. & 1928. & 2100. \\
\hline $\mathrm{A} 3 \mathrm{~B} 4=\mathrm{R} 12$ & 695.6 & 812.2 & 947.8 & $\overline{1} 150$. & 1318. & $\overline{1} 474$. & 1644. & $\overline{1} 795$. & 1925. & 2063. \\
\hline SEM & 6.69 & 11.67 & 18.11 & 19.32 & 25.85 & 27.09 & $\overline{2} 9.83$ & 38.00 & $\hat{4} 4.31$ & $\overline{5} 4.5$ \\
\hline Significance & NS & NS & NS & NS & NS & NS & NS & NS & NS & NS \\
\hline
\end{tabular}

With regard to the effect of dietary treatments on FCR, dietary corn levels had a significant effect $(\mathrm{P}<0.05)$ on FCR of growing rabbits during the periods of $6-7,7-8,9-10,11-12,12-13,13-14$ and from 14-15 weeks of age, but without significant effect during the whole experimental period (from 6-15 weeks) (Table 5).

Irrespective of the effect of dietary corn levels, FCR of rabbits fed control ration was significantly better $(\mathrm{P}<0.05)$ from 9-10 and from 14-15 weeks, while ration which supplemented with $0.05 \%$ enzume was the better at 13-14 weeks than the other supplements. The interaction between dietary corn levels and added supplements on FCR during the whole experimental period (from 6-15 weeks) showed that, the 


\section{Dorra et al.}

FCR of the growing rabbits were better $(\mathrm{P}<0.05)$ with rations R8, R9 and R11 (3.93, 4.07 and 4.04 g/d, respectively) as shown in Table (5).

Table (3): Effect of fed diets containing three levels of corn supplemented with enzyme or prebiotic or both on daily weight gain of growing rabbits from 6 to 15 weeks of age.

\begin{tabular}{|c|c|c|c|c|c|c|c|c|c|c|}
\hline \multirow{3}{*}{$\begin{array}{l}\text { Dietary } \\
\text { treatment }\end{array}$} & \multicolumn{10}{|c|}{ Daily weight gain (g) } \\
\hline & \multicolumn{10}{|c|}{ Week } \\
\hline & $6-7$ & $7-8$ & $8-9$ & $9-10$ & $10-11$ & $11-12$ & $12-13$ & $13-14$ & $14-15$ & $6-15$ \\
\hline \multicolumn{11}{|c|}{ Corn levels \% (A): } \\
\hline $5 \%(\mathrm{~A} 1)$ & $18.52^{\mathrm{a}}$ & $16.83^{\mathrm{b}}$ & 24.58 & $28.19^{\mathrm{a}}$ & 22.18 & 22.46 & 23.86 & 16.51 & 21.76 & 21.66 \\
\hline $10 \%(\mathrm{~A} 2)$ & $15.28^{\mathrm{b}}$ & $20.15^{\mathrm{a}}$ & 24.14 & $25.53^{\mathrm{b}}$ & 24.17 & 21.60 & 23.01 & 18.94 & 23.98 & 21.87 \\
\hline $15 \%(\mathrm{~A} 3)$ & $17.66^{\mathrm{a}}$ & $17.84^{\mathrm{a}}$ & 25.59 & $26.27^{\mathrm{a}}$ & 23.27 & 23.86 & 21.11 & 19.08 & 22.57 & 21.91 \\
\hline SEM & 0.69 & 0.82 & 0.99 & 0.78 & 0.75 & 0.72 & 0.95 & 0.85 & 0.99 & 0.43 \\
\hline Significance & $* *$ & $*$ & NS & $*$ & NS & NS & NS & NS & NS & NS \\
\hline \multicolumn{11}{|c|}{ Supplements \% (B): } \\
\hline $0.0 \% \quad(\mathrm{~B} 1)$ & 17.68 & 17.63 & $22.79^{\mathrm{b}}$ & $28.02^{\mathrm{a}}$ & 22.46 & $21.64^{\mathrm{b}}$ & 21.44 & 18.08 & 23.56 & 21.47 \\
\hline $0.05 \% \mathrm{E}(\mathrm{B} 2)$ & 16.39 & 18.81 & $24.42^{\mathrm{a}}$ & $26.31^{\mathrm{a}}$ & 23.66 & $21.71^{\mathrm{b}}$ & 21.80 & 19.10 & 23.43 & 21.74 \\
\hline $0.05 \% \mathrm{P}(\mathrm{B} 3)$ & 17.22 & 18.33 & $24.78^{\mathrm{a}}$ & $27.69^{a}$ & 23.99 & $22.62^{\mathrm{a}}$ & 24.00 & 16.93 & 21.44 & 22.01 \\
\hline $0.05 \% \mathrm{E}+0.05 \%$ & 17.32 & 18.31 & $27.10^{\mathrm{a}}$ & $24.62^{\mathrm{b}}$ & 22.72 & $24.60^{\mathrm{a}}$ & 23.39 & 18.59 & 22.67 & 22.02 \\
\hline$\overline{S E M}$ & 0.80 & 0.95 & 1.14 & 0.90 & 0.86 & 0.83 & 1.09 & 0.98 & 1.13 & 0.49 \\
\hline Significance & NS & NS & $*$ & $*$ & NS & $*$ & NS & NS & NS & NS \\
\hline \multicolumn{11}{|l|}{ AB Interaction: } \\
\hline $\mathrm{A} 1 \mathrm{~B} 1=\mathrm{R} 1$ & 17 & 14.60 & 23.27 & 30.33 & 20.60 & 19.23 & 22.30 & 16.03 & 23.93 & 20.80 \\
\hline $\mathrm{A} 1 \mathrm{~B} 2=\mathrm{R} 2$ & 19.93 & 18.10 & 25.63 & 27.60 & 22.23 & 21.47 & 23.73 & 18.27 & 24.43 & 22.37 \\
\hline $\mathrm{A} 1 \mathrm{~B} 3=\mathrm{R} 3$ & 18.50 & 18.33 & 22.93 & 26.90 & 22.93 & 24.40 & 25.83 & 15.03 & 21.10 & 21.77 \\
\hline $\mathrm{A} 1 \mathrm{~B} 4=\mathrm{R} 4$ & 18.63 & 16.27 & 26.50 & 28.03 & 22.97 & 24.80 & 23.57 & 16.70 & 17.63 & 21.70 \\
\hline $\mathrm{A} 2 \mathrm{~B} 1=\mathrm{R} 5$ & 16.13 & 20.40 & 22.07 & 26.83 & 23.27 & 20.70 & 21.97 & 18.33 & 23.67 & 21.47 \\
\hline $\mathrm{A} 2 \mathrm{~B} 2=\mathrm{R} 6$ & 12.47 & 20.97 & 23.90 & 26.27 & 26.03 & 20.60 & 20.87 & 19.83 & 22.93 & 21.57 \\
\hline $\mathrm{A} 2 \mathrm{~B} 3=\mathrm{R} 7$ & 15.87 & 19.93 & 24.73 & 27.10 & 24.50 & 20.43 & 24.20 & 16.97 & 22.40 & 21.77 \\
\hline $\mathrm{A} 2 \mathrm{~B} 4=\mathrm{R} 8$ & 16.67 & 19.30 & 25.87 & 21.90 & 22.87 & 24.67 & 25.00 & 20.63 & 26.93 & 22.67 \\
\hline $\mathrm{A} 3 \mathrm{~B} 1=\mathrm{R} 9$ & 19.90 & 17.90 & 23.03 & 27.00 & 23.50 & 25.00 & 20.07 & 19.87 & 23.07 & 22.13 \\
\hline $\mathrm{A} 3 \mathrm{~B} 2=\mathrm{R} 10$ & 16.77 & 17.37 & 23.73 & 25.07 & 22.70 & 23.07 & 20.80 & 19.20 & 22.93 & 21.30 \\
\hline $\mathrm{A} 3 \mathrm{~B} 3=\mathrm{R} 11$ & 17.30 & 16.73 & 26.67 & 29.07 & 24.53 & 23.03 & 21.97 & 18.80 & 24.50 & 22.50 \\
\hline $\mathrm{A} 3 \mathrm{~B} 4=\mathrm{R} 12$ & 16.67 & 19.37 & 29.93 & 23.93 & 22.33 & 24.33 & 21.60 & 18.43 & 19.77 & 21.70 \\
\hline SEM & 1.39 & 1.65 & 1.98 & 1.56 & 1.50 & 1.43 & 1.90 & 1.70 & 1.96 & 0.86 \\
\hline Significance & NS & NS & NS & NS & NS & NS & NS & NS & NS & NS \\
\hline
\end{tabular}

$a-b$ : For each of the main effects, means within the same column with different superscripts differ significantly $(P<0.05)$. SEM=standard error of means; NS: not significant; *: significant; **: highly significant.

The increase of DE concentration in high starch diets stimulated growth rate without affecting feed intake (Gutierrez et al., 2002). Amber et al. (2004) supplemented Lactobacillus acidophilus (probiotic) found a positive effect on average daily gain $(+9.6 \%)$ and negative effect on FCR $(-6.5 \%)$. Gidenne et al. (2002), reported that the improvement in live body weight and body weight gain of the rabbits fed enzymes may be due to the enhancing effect of enzymes on micro flora growth in gut and cecum as well as increase in volatile fatty acids (VFAs) production and organic matter digestibility.

The effect of feeding diets containing three levels of corn without or supplemented with enzyme or prebiotic or both on carcass characteristics of growing rabbits are illustrated in Table (6). Apart from the effect of feed which contains $5 \%$ corn a significant increased $(\mathrm{P}<0.05)$ on lungs weight than diets containing $10 \%$ or $15 \%$ corn. Apart from the effect of feed supplements, feeding the diet without any supplements increased $(\mathrm{P}<0.05)$ weights of lungs, while when supplemented diet with enzymes increased 
$(\mathrm{p}<0.05)$ liver weight and kidneys increased $(\mathrm{P}<0.05)$ with feeding on diets without supplements or with supplemented enzyme or enzyme plus prebiotic compared with those of the diet supplemented with prebiotic only. The effect of dietary interaction, showed addition of enzyme with diet containing $5 \%$ corn or diet containing $10 \%$ corn without supplements lead to a significant $(\mathrm{P}<0.05)$ increased in weight of viscera.

Table (4): Effect of fed diets containing three levels of corn supplemented with enzyme or prebiotic or both on daily feed intake (DFI) of growing rabbits from 6 to 15 weeks of age.

\begin{tabular}{|c|c|c|c|c|c|c|c|c|c|c|}
\hline \multirow{3}{*}{$\begin{array}{l}\text { Dietary } \\
\text { treatment }\end{array}$} & \multicolumn{10}{|c|}{ Daily feed intake (g) } \\
\hline & \multicolumn{10}{|c|}{ Week } \\
\hline & $6-7$ & $7-8$ & $8-9$ & $9-10$ & $10-11$ & $11-12$ & $12-13$ & $13-14$ & $14-15$ & $6-15$ \\
\hline \multicolumn{11}{|c|}{ Corn levels \% (A): } \\
\hline $5 \%(\mathrm{~A} 1)$ & 47.47 & 56.67 & 64.72 & 76.94 & 90.68 & 102.4 & 112.96 & 124.6 & 132.44 & 89.88 \\
\hline $10 \%(\mathrm{~A} 2)$ & 47.86 & 55.08 & 64.72 & 76.28 & 89.50 & $10 \hat{1.3}$ & 111.72 & $12 \overline{3} .1$ & 131.66 & 89.03 \\
\hline $15 \%(\mathrm{~A} 3)$ & 47.89 & 55.83 & 64.19 & 76.68 & 89.94 & 101.6 & 113.64 & 123.8 & 132.94 & 89.62 \\
\hline SEM & 0.24 & 0.50 & 0.69 & 0.84 & 1.1 & 1.23 & 1.15 & $1 . \overline{4} 0$ & 1.76 & 0.86 \\
\hline Significance & NS & NS & NS & NS & NS & NS & NS & NS & NS & NS \\
\hline \multicolumn{11}{|c|}{ Supplements \% (B): } \\
\hline $0.0 \% \quad$ (B1) & 47.82 & 55.55 & 64.37 & 75.59 & 89.78 & 100.8 & 111.41 & 121.89 & 130.70 & 88.67 \\
\hline $0.05 \% \mathrm{E}(\mathrm{B} 2)$ & 47.70 & 56.04 & 65.04 & 76.48 & 89.56 & $10 \overline{1} .1$ & 111.55 & 122.74 & 131.26 & 89.04 \\
\hline $0.05 \%$ P (B3) & 47.52 & 56.41 & 65.19 & 77.07 & 90.70 & 102.4 & 113.28 & 125.30 & 133.30 & 90.14 \\
\hline $0.05 \% \mathrm{E}+0.05 \%$ & 47.93 & 55.44 & 63.59 & 77.39 & 90.13 & $10 \hat{2} .7$ & 114.85 & 125.59 & 134.15 & 90.19 \\
\hline$\overline{\mathrm{SEM}}$ & 0.28 & 0.58 & 0.80 & 0.97 & 1.17 & $1 . \overline{4} 3$ & 1.33 & 1.62 & 2.04 & 0.99 \\
\hline Significance & NS & NS & NS & NS & NS & NS & NS & NS & NS & NS \\
\hline \multicolumn{11}{|l|}{ AB Interaction: } \\
\hline $\mathrm{A} 1 \mathrm{~B} 1=\mathrm{R} 1$ & 46.67 & 55.45 & 62.44 & 73.78 & 88.56 & 98.78 & 108.11 & 118.89 & 126.78 & 86.6 \\
\hline $\mathrm{A} 1 \mathrm{~B} 2=\mathrm{R} 2$ & 47.89 & 57.67 & 66.55 & 79.00 & 92.44 & 103.33 & 113.89 & 125.67 & 134.55 & 91.20 \\
\hline $\mathrm{A} 1 \mathrm{~B} 3=\mathrm{R} 3$ & 47.66 & 57.00 & 66.00 & 77.11 & 90.22 & 101.55 & 113.39 & 126.11 & 132.67 & 90.20 \\
\hline $\mathrm{A} 1 \mathrm{~B} 4=\mathrm{R} 4$ & 47.67 & 56.55 & 63.89 & 77.89 & 91.50 & 105.94 & 116.45 & 128.05 & 135.78 & 91.53 \\
\hline $\mathrm{A} 2 \mathrm{~B} 1=\mathrm{R} 5$ & 48.11 & 55.66 & 66.33 & 76.56 & 89.78 & 101.22 & 111.33 & 122.22 & 131.11 & 89.17 \\
\hline $\mathrm{A} 2 \mathrm{~B} 2=\mathrm{R} 6$ & 48.11 & 55.22 & 64.78 & 75.22 & 88.78 & 101.34 & 110.89 & 121.89 & 129.67 & 88.43 \\
\hline $\mathrm{A} 2 \mathrm{~B} 3=\mathrm{R} 7$ & 47.45 & 56.00 & 65.11 & 77.11 & 90.33 & 102.34 & 111.56 & 124.22 & 132.34 & 89.60 \\
\hline $\mathrm{A} 2 \mathrm{~B} 4=\mathrm{R} 8$ & 47.77 & 53.45 & 62.67 & 76.22 & 89.11 & 100.34 & 113.11 & 124.11 & 135.56 & 88.90 \\
\hline $\mathrm{A} 3 \mathrm{~B} 1=\mathrm{R} 9$ & 48.66 & 55.55 & 64.33 & 76.44 & 91.00 & 102.56 & 114.78 & 124.55 & 134.22 & 90.23 \\
\hline $\mathrm{A} 3 \mathrm{~B} 2=\mathrm{R} 10$ & 47.11 & 55.22 & 63.78 & 75.22 & 87.45 & 98.67 & 109.89 & 120.67 & 129.56 & 87.50 \\
\hline $\mathrm{A} 3 \mathrm{~B} 3=\mathrm{R} 11$ & 47.45 & 56.22 & 64.44 & 77.00 & 91.55 & 103.56 & 114.89 & 125.56 & 134.89 & 90.63 \\
\hline $\mathrm{A} 3 \mathrm{~B} 4=\mathrm{R} 12$ & 48.33 & 56.33 & 64.22 & 78.06 & 89.78 & 102.00 & 115.00 & 124.61 & 133.11 & 90.13 \\
\hline SEM & 0.484 & 1.001 & 1.39 & 1.676 & 2.032 & 2.474 & 2.309 & 2.809 & 3.529 & 1.719 \\
\hline Significance & NS & NS & NS & NS & NS & NS & NS & NS & NS & NS \\
\hline
\end{tabular}

The higher gut content in rabbits fed low starch diets depended on the higher dietary fiber concentration and explained the lower dressing percentage (Gidenne, 1992). Other traits of carcass and meat were non significant affected by dietary starch level. In fact rabbit carcass and meat quality substantially changed only when diet presented great nutrient excess or lack, being the ontogenetic factors (slaughter weight and age, sex, etc.) more effective (Xiccato, 1999).

The economic efficiency (\%) results (Table, 7) showed that feeding growing rabbits on ration which was containing $10 \%$ corn and supplemented with enzymes plus prebiotic (R8) or feeding diet contained $15 \%$ corn without supplements (R9) improved the feed efficiency was observed (102.84 and $102.46 \%$, 
respectively).

Table (5): Effect of fed diets containing three levels of corn supplemented with enzyme or prebiotic or both on feed conversion ratio (FCR) ratio of growing rabbits from 6 to 15 weeks of age.

\begin{tabular}{|c|c|c|c|c|c|c|c|c|c|c|}
\hline \multirow{3}{*}{$\begin{array}{l}\text { Dietary } \\
\text { treatment }\end{array}$} & \multicolumn{10}{|c|}{ Feed conversion ratio ( $\mathrm{g}$ feed: $\mathrm{g}$ gain) } \\
\hline & \multicolumn{10}{|c|}{ Week } \\
\hline & $6-7$ & $7-8$ & $8-9$ & $9-10$ & $10-11$ & $11-12$ & $12-13$ & $13-14$ & $14-15$ & $6-15$ \\
\hline \multicolumn{11}{|c|}{ Corn levels \% (A): } \\
\hline $5 \%(\mathrm{~A} 1)$ & $2.60^{\mathrm{b}}$ & $3.51^{\mathrm{a}}$ & 2.66 & $2.76^{\mathrm{b}}$ & 4.14 & $4.65^{\mathrm{ab}}$ & $4.83^{\mathrm{b}}$ & $7.70^{\mathrm{a}}$ & $6.25^{\mathrm{a}}$ & 4.17 \\
\hline $10 \%(\mathrm{~A} 2)$ & $3.22^{\mathrm{a}}$ & $2.77^{\mathrm{b}}$ & 2.78 & $3.02^{\mathrm{a}}$ & 3.75 & $4.74^{\mathrm{a}}$ & $4.94^{\mathrm{ab}}$ & $6.59^{\mathrm{b}}$ & $5.54^{\mathrm{b}}$ & 4.08 \\
\hline $15 \%(\mathrm{~A} 3)$ & $2.76^{\mathrm{b}}$ & $3.17^{\mathrm{ab}}$ & 2.55 & $2.95^{\mathrm{ab}}$ & 3.88 & $4.30^{\mathrm{b}}$ & $5.41^{\mathrm{a}}$ & $6.69^{\mathrm{b}}$ & $6.08^{\mathrm{ab}}$ & 4.09 \\
\hline SEM & 0.12 & 0.17 & 0.36 & 0.07 & 0.13 & 0.14 & 0.18 & 0.33 & 0.23 & 0.05 \\
\hline Significance & $* *$ & $*$ & NS & $*$ & NS & $*$ & $*$ & $*$ & $*$ & NS \\
\hline \multicolumn{11}{|c|}{ Supplements \% (B): } \\
\hline $0.0 \% \quad(\mathrm{~B} 1)$ & 2.76 & 3.26 & 2.93 & $2.72^{\mathrm{b}}$ & 4.05 & 4.73 & 5.26 & $6.81^{\mathrm{ab}}$ & $5.57^{\mathrm{b}}$ & 4.13 \\
\hline $0.05 \% \mathrm{E}(\mathrm{B} 2)$ & 3.08 & 3.04 & 2.68 & $2.94^{\mathrm{ab}}$ & 3.82 & 4.70 & 5.21 & $6.55^{\mathrm{b}}$ & $5.70^{\mathrm{b}}$ & 4.11 \\
\hline $0.05 \% \mathrm{P}(\mathrm{B} 3)$ & 2.79 & 3.11 & $2.68^{\mathrm{b}}$ & $2.80^{\mathrm{b}}$ & 3.84 & 4.62 & 4.80 & $7.76^{\mathrm{a}}$ & $6.02^{\mathrm{ab}}$ & 4.10 \\
\hline $0.05 \% \mathrm{E}+0.05 \%$ & 2.81 & 3.19 & $2.37^{\mathrm{b}}$ & $3.18^{\mathrm{a}}$ & 3.99 & 4.20 & 4.96 & $6.86^{\mathrm{ab}}$ & $6.53^{\mathrm{a}}$ & 4.10 \\
\hline$\overline{\mathrm{SEM}}$ & 0.14 & 0.20 & 0.16 & 0.08 & 0.15 & 0.17 & 0.21 & 0.38 & 0.26 & 0.06 \\
\hline Significance & NS & NS & NS & $* *$ & NS & NS & NS & $*$ & $*$ & NS \\
\hline \multicolumn{11}{|l|}{ AB Interaction: } \\
\hline $\mathrm{A} 1 \mathrm{~B} 1=\mathrm{R} 1$ & 2.77 & 3.88 & 2.69 & 2.45 & 4.36 & 5.19 & 4.93 & 7.43 & 5.31 & 4.17 \\
\hline $\mathrm{A} 1 \mathrm{~B} 2=\mathrm{R} 2$ & 2.45 & 3.23 & 2.59 & 2.94 & 4.18 & 4.83 & 4.97 & 7.06 & 5.58 & 4.11 \\
\hline $\mathrm{A} 1 \mathrm{~B} 3=\mathrm{R} 3$ & 2.61 & 3.11 & 2.94 & 2.88 & 4.01 & 4.30 & 4.45 & 8.57 & 6.37 & 4.16 \\
\hline $\mathrm{A} 1 \mathrm{~B} 4=\mathrm{R} 4$ & 2.58 & 3.81 & 2.42 & 2.78 & 4.00 & 4.29 & 4.94 & 7.74 & 7.72 & 4.23 \\
\hline $\mathrm{A} 2 \mathrm{~B} 1=\mathrm{R} 5$ & 3.05 & 2.80 & 3.26 & 2.87 & 3.89 & 4.89 & 5.09 & 6.71 & 5.55 & 4.16 \\
\hline $\mathrm{A} 2 \mathrm{~B} 2=\mathrm{R} 6$ & 3.92 & 2.64 & 2.72 & 2.87 & 3.43 & 4.93 & 5.36 & 6.17 & 5.69 & 4.11 \\
\hline $\mathrm{A} 2 \mathrm{~B} 3=\mathrm{R} 7$ & 2.99 & 2.83 & 2.67 & 2.85 & 3.75 & 5.04 & 4.72 & 7.45 & 5.94 & 4.11 \\
\hline $\mathrm{A} 2 \mathrm{~B} 4=\mathrm{R} 8$ & 2.92 & 2.82 & 2.47 & 3.49 & 3.93 & 4.09 & 4.57 & 6.04 & 4.96 & 3.93 \\
\hline $\mathrm{A} 3 \mathrm{~B} 1=\mathrm{R} 9$ & 2.47 & 3.10 & 2.83 & 2.84 & 3.89 & 4.11 & 5.76 & 6.29 & 5.86 & 4.07 \\
\hline $\mathrm{A} 3 \mathrm{~B} 2=\mathrm{R} 10$ & 2.85 & 3.27 & 2.72 & 3.01 & 3.85 & 4.35 & 5.29 & 6.43 & 5.83 & 4.11 \\
\hline A3B3= R11 & 2.77 & 3.39 & 2.42 & 2.66 & 3.77 & 4.53 & 5.23 & 7.24 & 5.75 & 4.04 \\
\hline $\mathrm{A} 3 \mathrm{~B} 4=\mathrm{R} 12$ & 2.93 & 2.93 & 2.22 & 3.27 & 4.03 & 4.21 & 5.37 & 6.79 & 6.90 & 4.15 \\
\hline SEM & 0.241 & 0.346 & 0.272 & 0.147 & 0.259 & 0.286 & 0.358 & 0.652 & 0.455 & 0.102 \\
\hline Significance & NS & NS & NS & NS & NS & NS & NS & NS & $*$ & $*$ \\
\hline
\end{tabular}
$a-b$ : For each of the main effects, means within the same column with different superscripts differ significantly $(P \unlhd 0.05)$. SEM=standard error of means; NS: not significant; *: significant; **: highly significant. 
Table (6): Effect of fed diets containing three levels of corn supplemented with enzyme or prebiotic or both on some carcass characteristics of 15 -week-old growing rabbits.

\begin{tabular}{|c|c|c|c|c|c|c|c|c|c|}
\hline $\begin{array}{l}\text { Dietary } \\
\text { treatment }\end{array}$ & LBW (g) & $\begin{array}{c}\text { Dressing } \\
(\%)\end{array}$ & $\begin{array}{l}\text { Skin wt. } \\
\text { (g) }\end{array}$ & $\begin{array}{l}\text { Lungs } \\
\text { wt. (g) }\end{array}$ & $\begin{array}{l}\text { Viscera } \\
\text { wt. (g) }\end{array}$ & $\begin{array}{l}\text { Liver wt. } \\
\text { (g) }\end{array}$ & $\begin{array}{l}\text { Heart } \\
\text { wt.(g) }\end{array}$ & $\begin{array}{l}\text { Kidneys } \\
\text { wt. (g) }\end{array}$ & $\begin{array}{c}\text { Total } \\
\text { dressin } \\
\mathrm{g}(\%)\end{array}$ \\
\hline \multicolumn{10}{|c|}{ Corn levels \% (A): } \\
\hline $5 \%(\mathrm{~A} 1)$ & 2146.3 & 57.48 & 382.1 & $18.61^{\mathrm{a}}$ & 347.9 & 70.23 & 5.82 & 17.47 & 61.83 \\
\hline $10 \%(\mathrm{~A} 2)$ & 2202.9 & 55.96 & 387.9 & $16.38^{\mathrm{b}}$ & 361.3 & 74.8 & 5.73 & 17.09 & 60.38 \\
\hline $15 \%(\mathrm{~A} 3)$ & 2165.4 & 57.07 & 395.4 & $14.84^{\mathrm{c}}$ & 322.9 & 67.45 & 6.34 & 17.88 & 61.31 \\
\hline SEM & 51.90 & 0.58 & 11.32 & 0.47 & 12.96 & 3.42 & 0.40 & 0.62 & 0.55 \\
\hline Significance & NS & NS & NS & $* *$ & NS & NS & NS & NS & NS \\
\hline \multicolumn{10}{|c|}{ Supplements \% (B): } \\
\hline $0.0 \% \quad$ (B1) & 2168.3 & 56.42 & 393.89 & $17.89^{\mathrm{a}}$ & 348.3 & $69.98^{\mathrm{ab}}$ & 5.2 & $17.5^{\mathrm{a}}$ & 60.69 \\
\hline $0.05 \% \mathrm{E}(\mathrm{B} 2)$ & 2181.1 & 56.30 & 378.3 & $16.17^{\mathrm{bc}}$ & 366.1 & $80.23^{\mathrm{a}}$ & 6.21 & $19.1^{\mathrm{a}}$ & 61.14 \\
\hline $0.05 \% \mathrm{P}(\mathrm{B} 3)$ & 2171.1 & 57.52 & 381.7 & $15.3^{\mathrm{c}}$ & 332.8 & $68.49^{\mathrm{ab}}$ & 6.49 & $15.4^{\mathrm{b}}$ & 61.67 \\
\hline $0.05 \% \mathrm{E}+0.05 \%$ & 2165.6 & 57.1 & 400.00 & $17.1^{\mathrm{ab}}$ & 328.9 & $64.61^{\mathrm{b}}$ & 5.96 & $17.9^{\mathrm{a}}$ & 61.18 \\
\hline$\overline{\mathrm{SEM}}$ & 59.94 & 0.67 & 13.07 & 0.54 & 14.96 & 3.95 & 0.46 & 0.71 & 0.64 \\
\hline Significance & NS & NS & NS & $* *$ & NS & $*$ & NS & $* *$ & NS \\
\hline \multicolumn{10}{|l|}{ AB Interaction: } \\
\hline $\mathrm{A} 1 \mathrm{~B} 1=\mathrm{R} 1$ & 2070.0 & 56.41 & 381.7 & 21.1 & 333.3 & 66.83 & 4.70 & 18.00 & 60.73 \\
\hline $\mathrm{A} 1 \mathrm{~B} 2=\mathrm{R} 2$ & 2286.7 & 56.89 & 398.3 & 18.07 & 446.7 & 78.53 & 6.07 & 19.77 & 61.45 \\
\hline $\mathrm{A} 1 \mathrm{~B} 3=\mathrm{R} 3$ & 2140.0 & 58.21 & 361.7 & 16.30 & 313.3 & 78.30 & 5.87 & 14.30 & 62.79 \\
\hline $\mathrm{A} 1 \mathrm{~B} 4=\mathrm{R} 4$ & 2088.3 & 58.43 & 386.7 & 18.97 & 298.3 & 57.23 & 6.63 & 17.80 & 62.35 \\
\hline $\mathrm{A} 2 \mathrm{~B} 1=\mathrm{R} 5$ & 2291.6 & 55.26 & 386.7 & 17.47 & 410.0 & 81.77 & 5.40 & 17.40 & 59.83 \\
\hline $\mathrm{A} 2 \mathrm{~B} 2=\mathrm{R} 6$ & 2131.7 & 55.31 & 371.7 & 16.53 & 331.7 & 82.63 & 5.70 & 18.10 & 60.30 \\
\hline $\mathrm{A} 2 \mathrm{~B} 3=\mathrm{R} 7$ & 2166.7 & 56.41 & 383.3 & 15.50 & 355.0 & 63.57 & 6.77 & 15.27 & 60.34 \\
\hline $\mathrm{A} 2 \mathrm{~B} 4=\mathrm{R} 8$ & 2221.7 & 56.84 & 410.0 & 16.00 & 348.3 & 71.23 & 5.07 & 17.60 & 61.05 \\
\hline $\mathrm{A} 3 \mathrm{~B} 1=\mathrm{R} 9$ & 2143.3 & 57.61 & 413.3 & 15.10 & 301.7 & 61.33 & 5.50 & 17.10 & 61.53 \\
\hline $\mathrm{A} 3 \mathrm{~B} 2=\mathrm{R} 10$ & 2125.0 & 56.69 & 365.0 & 13.90 & 320.0 & 79.53 & 6.87 & 19.43 & 61.68 \\
\hline $\mathrm{A} 3 \mathrm{~B} 3=\mathrm{R} 11$ & 2206.7 & 57.93 & 400.0 & 14.03 & 330.0 & 63.60 & 6.83 & 16.63 & 61.87 \\
\hline $\mathrm{A} 3 \mathrm{~B} 4=\mathrm{R} 12$ & 2186.7 & 56.03 & 403.3 & 16.33 & 340.0 & 65.37 & 5.17 & 18.37 & 60.14 \\
\hline SEM & 103.81 & 1.16 & 22.64 & 0.94 & 25.92 & 6.84 & 0.79 & 1.24 & 1.11 \\
\hline Significance & NS & NS & NS & NS & $* *$ & NS & NS & NS & NS \\
\hline
\end{tabular}

$a-b$ : For each of the main effects, means within the same column with different superscripts differ significantly $(P \unlhd 0.05)$. SEM=standard error of means; NS: not significant; *: significant; **: highly significant.

Total dressing percentage $=($ carcass yield + edible organs/live body weight $) X 100$. 
Table (7): Effect of fed diets containing three levels of corn supplemented with enzyme or prebiotic or both on economic efficiency of growing rabbits from 6 to 15 weeks of age.

\begin{tabular}{lcccccccccccc}
\hline Item & R1 & R2 & R3 & R4 & R5 & R6 & R7 & R8 & R9 & R10 & R11 & R12 \\
\hline $\begin{array}{l}\text { DMI (g/d) } \\
\text { Mean gain }\end{array}$ & 86.60 & 91.20 & 90.2 & 91.5 & 89.17 & 88.4 & 89.6 & 88.90 & 90.23 & 87.5 & 90.63 & 90.1 \\
$\begin{array}{l}\text { (g/d) } \\
\text { Feed cost }\end{array}$ & 20.80 & 22.47 & 21.8 & 21.7 & 21.57 & 21.6 & 21.8 & 22.77 & 22.13 & 21.3 & 22.50 & 21.7 \\
$($ LE) & 0.364 & 0.390 & 0.38 & 0.40 & 0.375 & 0.37 & 0.38 & 0.391 & 0.383 & 0.37 & 0.394 & 0.39 \\
$\begin{array}{l}\text { Meat price } \\
(\text { LE) }\end{array}$ & 0.728 & 0.783 & 0.76 & 0.75 & 0.751 & 0.75 & 0.76 & 0.793 & 0.775 & 0.74 & 0.788 & 0.75 \\
$\begin{array}{l}\text { Net } \\
\text { revnue (LE) }\end{array}$ & 0.364 & 0.393 & 0.37 & 0.35 & 0.376 & 0.37 & 0.37 & 0.402 & 0.392 & 0.36 & 0.394 & 0.36 \\
$\begin{array}{l}\text { Econ. Effic. } \\
(\%)\end{array}$ & 100.0 & 100.8 & 95.9 & 88.8 & 100.3 & 99.2 & 96.9 & 102.8 & 102.4 & 97.4 & 100.0 & 90.2 \\
\hline
\end{tabular}

\section{CONCLUSION}

Using corn in the rations of rabbits replacement of barley had no effect on growth performance and sanitary risk. Also, the using exogenous enzymes with prebiotics were slightly enhancing the growth performance and economic efficiency especially in the post-weaning period.

\section{REFERENCES}

Abo El-Maaty, Hayam M. A.; E. Abo Egla M. Qota and Sh. M. El-Desouky (2014). Performance and economical of growing NewZealand Whit rabbits fed cucumber (Cucumis sativus L.) vines straw without or with some feed additives under Egyptian conditions. Egypt. Poul. Sci., 14 (II): 413.

Amber, K. H., H. M. Yakent and Rawya S. Hamed (2004). Effect of feeding diets containing yucca extract or probiotic on growth, digestibility, nitrogen balance and caecal microbial activity of growing New Zealand white rabbit. PP 737-741 in proceeding $8^{\text {th }}$ World Rabbit Congress.

Campell , G, L. and M.R. Bedford (1992). Enzyme applications for monogastric feeds : A review . Can. J. Amin. Sci., 72: 449.

Cowan, W.D.; A. Korsbak; T. Hastrup and B.P. Rasmussen (1996). Influence of added microbial enzymes on energy and protein availability of selected feed ingredients. Anim. Feed Sci. Technol. 60: 311.

Danicke, S., W. Vahjen, O. Simon and H. Jeroch (1999). Effect of dietary fat type and xylanase supplementation to rye-based broiler diets on selected bacterial groups adhering to the intestinal epithelium. on transit time of feed, and on nutrient digestibility. Poult Sci. 78(9):1292.

De Blac, C. and J. Wiseman (2003). The Nutrition of the Rabbit. CABI publishing, Oxon, UK.

Duncan, D. B. (1955). Multiple Range and Multiple F Test. Biometrics, 11: 1 - 24.

Falcao - e- Cunha, L., L. Castro - Solla; L. Maertens, M. Marounek, V. Pinheiro; J. Freire and J.L. Mourao (2007). Alternatives to antibiotic growth promoters in rabbit feeding: a review World Rabbit Science, $15,127$.

Garcia , A.I., J. Garcia, E. Corrent, S. Chamorro; P. Garcia-Rebollar; J.C. De Blas and R. Carabano (2005). Effect of rabbit age, type of protein and feed enzyme addition on the apparent dry matter and crude protein digestibility of rabbit feed. In Proc. Ilemes Journees de la Recherche Cunicole , 2005 November, Paris , France, 197.

Gibson, G.R. and M.B. Roberfroid (1995). Dietary modulation of the human colonic micro-biota: 
introducing the concept of prebiotics. Journal of Nutrition 125, 1401.

Gidenne, T. (1992). Effect of fiber level, particle size and adaptation period on digestibility and rate of passage as measured at the ileum and in the faeces in the adult rabbit. Br. J. Nutr., 67, 133.

Gidenne, T.; R. Carabano; J. Garcia and C.D. Blas (2010). Fiber digestion. In: De Blas , C., Wiseman , J. (Ed.), Nutrition of the rabbit . CABI , P. 179.

Gidenne, T.; N. Jehhl; M. Segura and B. Michalet-Doreau (2002). Microbial activity in the caecum of the rabbit around weaning impact of a dietary fiber deficiency and of intake level. Animal Feed Science and Technology 99: 107.

Gutierrez, I.; A. Espinosa; J. Garcia; R. Carabana and C. De Blas (2002). Effect of levels of starch, fiber and lactose on digestion and growth performance of early weaned rabbits. Journal of Animal Science 80, 1029.

Nizza, A.and G. Moniello (2000). Meat quality and caecal content characteristics of rabbit according to dietary content and botanical origin of starch. World Rabbit Science, 8,3 .

NRC Nutritional Research Council (1977). Nutrient requirement of rabbits. Nutritional Academic of Sciences, Washington, DC., USA.

Remois, G., P. Lafargue-Hauret and H. Rouillere (1996). Effect of amylases supplementation in rabbit feed on growth performance. In: Lebas, F. (ed.) Proceedings of the 6th World Rabbit Congress, Toulouse. 1996. Vol. 1. 289.

SAS (2000). Statistical Analysis System / STAT User's Guide, release 6.03. Ed., SAS Institute . Inc., Cary, Nc. USA, PP. 125.

Shanmuganathan, T.; K. Samarasinghe and C. Wenk (2004).Supplelemental enzymes, yeast culture and effective micro-organism culture to enhance the performance of rabbits fed diets containing high levels of rice bran. Asian - Aust. J. Amin. Sci. 17, 5:678.

Taylor, M.; S.L. Godwin; N. Ekhator and R.J. Coppings (1989). Meat to bone ratio, cooking losses and plate waste of meat from fryer rabbits. J.Appl. Rabbit Res. 12: 106.

Williams, P.E.V. and C.J. Newbold (1990). The effects of novel microorganisms on rumen fermentation and ruminant productivity in : (Ed. D.J.A. Cole and W. Haresign). Recent Advances in Animal Nutrition, Butterwoths, London .

Xiccato, G. (1999). Feeding and meat quality in rabbits: a review. World Rabbit Sci., 7, 75.

Xiccato, G.; M. E. Cossu; A. Trocino and P. I. Queaque (1998). Influence du rapport amidon/fibre et de l'addition de graise en post-sevrage sur la digestión, les performances zootechniques et la qualite' bouche're du lapin, in Proc. $7^{\text {emes }}$ Jpurne'es de la Recherche Cunicole, Lyon, France May, 154. 


\title{
Dorra et al.
}

تأثير التغذيه على مستويات مختلفة من الأذرة بدون أو بإضافة مخلوط الأنزيمات أو البريبيوتك على النمو والكفاعة الإقتصادية في علائق الارانب النامية

\author{
ترك محم إبراهيم دره و هيام محمل أبو المعاطي 1 و جيهان محم المغازي² و رنا حسين الثافعي عيد²

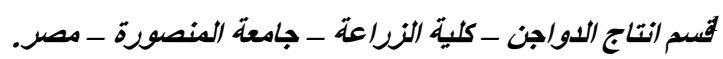

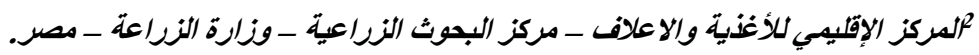

استخدم عدد 108 أرنب (نيوزيلندى أبيض) عمر 6 أسابيع حديثى الفطام لار اسة تأثير التغذية على علائق تحتوى على ثلاث مستويات

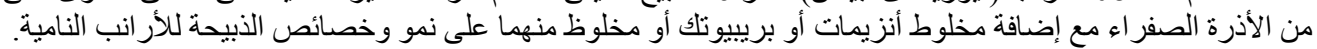

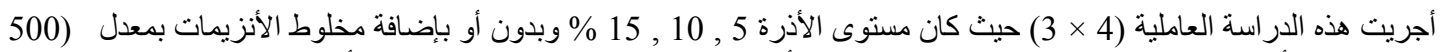

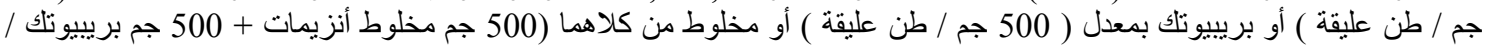
طن عليقة ) / طن عليقة.

وز عت الأر انب عثو ائيا ( 12 مجمو عة تجرييية) حيث تحتوى كل منها على 3 مكررات بالتنساوى فى الجاميع وكل مكررة تحتوى

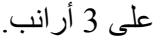

تم تغذية كل مجموعة على أى من العلائق التجريبية خلال الفترة من 6 - 15 أسبوع من عمر الأرانب وكان الغذاء والماء أمام

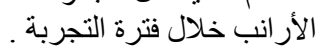

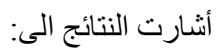

زيادة وزن الأرانب معنويا عند عمر 7 أسابيع بالتغذية على علائق تحتوى على 5 , 15 م \% أذرة مقارنة بالتغذية على العليقة

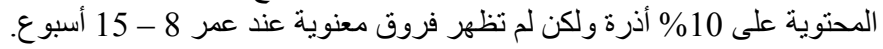

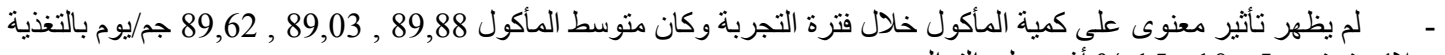
على علائق تحنوى 5 , 10 , 15 \% أذرة على التو الى الى خلي.

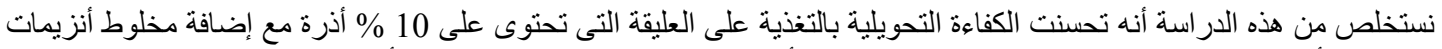
مع البريبيوتك أو التخذية على العليقة التى تحتوى على 15 \% أذرة بدون إضافات وكانت الكفاءة الأقتصادية 102,8 , 102,4 \% على الثى 